\title{
Daphnoretin induces reactive oxygen species-mediated apoptosis in melanoma cells
}

\author{
HUI WANG ${ }^{1 *}$, XUE HU $^{2 *}$, MINJING $\mathrm{LI}^{2}$, ZHAOHAI PAN ${ }^{2}$, DEFANG $\mathrm{LI}^{2}$ and QIUSHENG ZHENG ${ }^{1,2}$ \\ ${ }^{1}$ Key Laboratory of Xinjiang Phytomedicine Resource and Utilization, Ministry of Education, School of Pharmacy, \\ Shihezi University, Shihezi, Xinjiang 832003; ${ }^{2}$ Yantai Key Laboratory of Pharmacology of \\ Traditional Chinese Medicine in Tumor Metabolism, School of Integrated Traditional Chinese and \\ Western Medicine, Binzhou Medical University, Yantai, Shandong 264003, P.R. China
}

Received October 14, 2020; Accepted February 25, 2021

DOI: $10.3892 / 01.2021 .12714$

\begin{abstract}
Research suggests that daphnoretin exhibits a diverse array of antitumor mechanisms and pharmacological activities. However, there is no definitive explanation for the antitumor mechanisms of daphnoretin in malignant melanoma. In the present study, MTT and colony formation assays demonstrated that daphnoretin significantly inhibited the proliferation of melanoma A375 and B16 cells. Following treatment with daphnoretin, apoptotic bodies were observed in A375 and B16 cells via Hoechst 33258 staining. Furthermore, western blot analysis revealed that the apoptosis-related proteins cleaved caspase-3, cleaved caspase-9, Bax, cytochrome $c$ and apoptotic protease-activating factor 1 were significantly upregulated, while the expression levels of caspase-3, caspase-9 and Bcl-2 were downregulated in A375 and B16 cells. Flow cytometry and fluorescence microscopy revealed that daphnoretin induced higher levels of reactive oxygen species (ROS). Therefore, the results of the present study indicated that daphnoretin induced ROS-mediated mitochondria apoptosis in human (A375) and murine (B16) malignant melanoma cells.
\end{abstract}

Correspondence to: Professor Defang Li, Yantai Key Laboratory of Pharmacology of Traditional Chinese Medicine in Tumor Metabolism, School of Integrated Traditional Chinese and Western Medicine, Binzhou Medical University, Room A604, 346 Guanhai Road, Yantai, Shandong 264003, P.R. China

E-mail: lidefang@163.com

Professor Qiusheng Zheng, Key Laboratory of Xinjiang Phytomedicine Resource and Utilization, Ministry of Education, School of Pharmacy, Shihezi University, Room A401, New Medicine Building, 252 North Second Road, Shihezi, Xinjiang 832003, P.R. China

E-mail: zqsyt@sohu.com

${ }^{*}$ Contributed equally

Key words: daphnoretin, apoptosis, A375 cells, B16 cells

\section{Introduction}

Melanoma, also known as malignant melanoma, is characterized by the abnormal proliferation of melanocytes in the skin (1). Melanoma accounts for $\sim 2 \%$ of all skin cancer cases, but due to its strong invasiveness, causes the majority of skin cancer-associated deaths (2). The incidence rate and degree of malignancy in melanoma are high, with seriously impacts on human health (3). Melanoma is less common than other types of skin cancer, but more likely to invade nearby tissues and metastasize to other regions of the body (4). Furthermore, the rate of melanoma occurrence has increased considerably over the past few decades (5). Currently, surgery, chemotherapy and radiotherapy are the primary treatment options (6), and in the early stages of melanoma, the lesion can be successfully resected (7). Although clinical treatments are becoming increasingly more refined, a number of disadvantages remain, including the various side effects of radiotherapy and chemotherapy. Due to the frequent emergence of drug resistance, currently-available chemotherapeutics do not effectively treat patients with advanced melanoma.

As a result of good efficacy, mild side effects, low toxicity and its numerous targets, Traditional Chinese Medicine has been used to treat various cancer types (8). Consequently, the identification of effective anti-melanoma drugs from Chinese plant medicine is of great clinical significance. Traditional Chinese Medicine comprises complex components with low drug resistance (9), and leading antitumor components with novel structures and significant pharmacological activity have been confirmed $(10,11)$. Some of these components act on multiple targets simultaneously or cooperatively, making it possible to screen multi-target therapeutic drugs that meet clinical requirements $(12,13)$. Daphnoretin (14), which is extracted from Wikstroemia indica (15), has been proven to exert significant antitumor effects. Nevertheless, the mechanism of its antitumor properties in melanoma remains unclear, thus prompted the evaluation of daphnoretin treatment in the present study.

To determine the possible intrinsic mechanism of daphnoretin-induced melanoma cell apoptosis, the antiproliferative effect of daphnoretin on melanoma A375 and B16 cells was examined using MTT and colony formation assays. Subsequently, the apoptotic rate and the levels 
of apoptosis- associated proteins were determined by Hoechst 33258 staining and western blotting. In addition, the ROS levels in daphnoretin-treated A375 and B16 cells were detected using DCFH-DA probes. These findings may promote further research into novel treatments for melanoma.

\section{Materials and methods}

Cell culture and reagents. Human A375 and murine B16 malignant melanoma cells were purchased from the cell banks of the Chinese Academy of Sciences. Daphnoretin (cat no. X-051-191008; purity, $\geq 98 \%$ ) was purchased from Chengdu Herbpurify Co., Ltd. DMEM high-glucose culture medium was procured from HyClone (Cytiva), and supplemented with $10 \%$ FBS and $1 \%$ penicillin-streptomycin (Beijing Solarbio Science \& Technology Co., Ltd.). The cells were maintained in a humidified incubator at $37^{\circ} \mathrm{C}\left(5 \% \mathrm{CO}_{2}\right)$.

MTT assay. Cells were seeded into 96-well plates at a density of $1 \times 10^{5}$ cells $/ \mathrm{ml}$ and cultured for $24 \mathrm{~h}$, after which different concentrations of daphnoretin $(0,10,20,30,40$, $50,60,70$ and $80 \mu \mathrm{g} / \mathrm{ml}$ ) were added for a further $24 \mathrm{~h}$. Next, $20 \mu \mathrm{l} \mathrm{MTT}(5 \mathrm{mg} / \mathrm{ml})$ was added to each well and the cells were incubated for an additional $4 \mathrm{~h}$ before the media were discarded. Then, $150 \mu 1$ DMSO was added, and the plates were agitated for $10 \mathrm{~min}$ at room temperature. The absorbance value was determined using a microplate reader (Infinite 200 Pro; Tecan Group, Ltd.) at $490 \mathrm{~nm}$ (16).

Colony formation assay. Cells in the logarithmic phase were harvested with $0.25 \%$ trypsin and homogenized. The cells were resuspended in DMEM medium with 10\% FBS and then seeded into 6-well plates at 200 cells/well. Once adherent proliferation was established, the cells were treated with different doses of daphnoretin $(0,20,40$ and $60 \mu \mathrm{g} / \mathrm{ml})$ and incubated for $24 \mathrm{~h}$. On the second day, the media were replaced, and the cells were continuously cultured in a $37^{\circ} \mathrm{C}$ $\left(5 \% \mathrm{CO}_{2}\right)$; the media were then replaced once every three days for 2-3 weeks. The cells were fixed with $4 \%$ paraformaldehyde solution (Beijing Solarbio Science \& Technology Co., Ltd.) for $10 \mathrm{~min}$ at $4{ }^{\circ} \mathrm{C}$, and then stained with $1 \%$ crystal violet for $10 \mathrm{~min}$ at room temperature (both Beijing Solarbio Science $\&$ Technology Co., Ltd.). The cells were observed under a fluorescence microscope (DMI3000B, Leica Microsystems $\mathrm{GmbH}$ ) and images were captured.

Hoechst 33258 staining. To observed changes in morphology, cells were seeded into 6 -well plates $\left(2 \times 10^{5}\right.$ cells $\left./ \mathrm{ml}\right)$ and incubated for $24 \mathrm{~h}$, after which different concentrations of daphnoretin $(0,20,40$ and $60 \mu \mathrm{g} / \mathrm{ml})$ were added. After $24 \mathrm{~h}$, $4 \%$ paraformaldehyde solution was added at $4^{\circ} \mathrm{C}$. After $10 \mathrm{~min}$, the cells were washed twice with PBS and stained with Hoechst 33258 for $10 \mathrm{~min}$ at room temperature. Images were then captured using a fluorescence microscope (17).

Flow cytometric apoptosis analysis. Apoptosis was detected by flow cytometry. The cells were seeded into 6-well plates $\left(2 \times 10^{5}\right.$ cells $\left./ \mathrm{ml}\right)$ and incubated for $24 \mathrm{~h}$. Then, daphnoretin was added at different concentrations $(0,20,40$ and $60 \mu \mathrm{g} / \mathrm{ml})$. The cells were collected after $24 \mathrm{~h}$, and $10 \mu \mathrm{l}$ propidium iodide was added for $10 \mathrm{~min}$, followed by $5 \mu \mathrm{l}$ Annexin V-FITC for a further $5 \mathrm{~min}$ (both at room temperature). Flow cytometry was performed using a FACSCanto II flow cytometer (Becton, Dickinson and Company) (18). The apoptosis rate was analyzed using FACSDiva 6.1.3 software (Becton, Dickinson and Company).

ROS detection. Cells were seeded into 6-well plates at a density of $1.8 \times 10^{5}$ cells $/ \mathrm{ml}$, and then incubated for $24 \mathrm{~h}$. Different concentrations of daphnoretin were administered $(0,20,40$ and $60 \mu \mathrm{g} / \mathrm{ml})$ for $24 \mathrm{~h}$, after which the cells were digested with $0.25 \%$ trypsin (Beijing Solarbio Science \& Technology Co., Ltd.). DCFH-DA (Beijing Solarbio Science $\&$ Technology Co., Ltd.) was diluted with serum-free medium $(1: 1,000)$ to a final concentration of $10 \mu \mathrm{M}$, and $1 \mathrm{ml}$ was added per well prior to incubation at $37^{\circ} \mathrm{C}$ for $20 \mathrm{~min}$. After washing three times with serum-free medium, the cells were analyzed by flow cytometry or photographed under a fluorescence microscope.

Western blot analysis. Cells were seeded into 100-mm cell culture dishes with daphnoretin administered $(0$, 20,40 and $60 \mu \mathrm{g} / \mathrm{ml}$ ) and incubated for $24 \mathrm{~h}$. The cells were then collected and washed twice with PBS. RIPA buffer (cat. no. R0010; Beijing Solarbio Science \& Technology Co., Ltd.) was added to lyse the cells, and the mixture was kept on ice for $30 \mathrm{~min}$. The lysate was then centrifuged at $13,000 \mathrm{x} \mathrm{g}$ for $4 \mathrm{~min}$ at $4^{\circ} \mathrm{C}$, and the protein concentration was measured using BCA protein assay kit, and equalized between samples using $4 \mathrm{X}$ loading buffer. The mass of protein loaded per well was $80 \mu \mathrm{g}$. The samples were run on a $12 \%$ SDS-PAGE gel, transferred to PVDF membranes (EMD Millipore) and then blocked with $5 \%$ milk for $1 \mathrm{~h}$ at room temperature. Diluted primary antibodies against the following targets were then added overnight at $4^{\circ} \mathrm{C}$ : $\beta$-actin, (cat. no. TA-09; 1:2,000; OriGene Technologies, Inc.); caspase-3 (cat. no. ab13847; 1:1,000; Abcam); cleaved caspase-3 (cat. no. 9661S; 1:1,000; Cell Signaling Technology, Inc.); caspase-9 (cat. no. Ab202068; 1:2,000; Abcam); cleaved caspase-9 (cat. no. 20750S; 1:1,000; Cell Signaling Technology, Inc.); apoptotic protease-activating factor 1 (Apaf-1; cat no. 5088S; 1:1,000; Cell Signaling Technology, Inc.); cytochrome $c$ (cat. no. ab110325; 1:2,000; Abcam); Bcl-2 (cat. no. ab196495; 1:1,000, Abcam); Bax (cat. no. ab182734; 1:1,000; Abcam); PI3K (cat. no. 60225-1; 1:5,000, ProteinTech Group, Inc.); phospho (p-)PI3K (cat. no. ab182651; 1:500; Abcam); Akt (cat. no. 9272S; 1:1,000; Cell Signaling Technology, Inc.); and p-Akt (cat. no. 4060S; 1:2,000; Cell Signaling Technology, Inc.). The membranes were washed four times for 5 min each (with 1X TBST on a shaking platform), and then diluted secondary antibodies against the following targets were added overnight at room temperature: Peroxidase-conjugated affinipure goat anti-mouse IgG (cat. no. ZB-2305; 1:30,000; OriGene Technologies, Inc.) and goat anti-rabbit IgG (cat. no. ab6721; 1:20,000; Abcam). Membranes were washed four times for $5 \mathrm{~min}$ with 1X TBST. Tweezers were used to move each membrane to a culture dish, and ECL chemiluminescence working buffer (cat. no. 34580; Thermo Fisher Scientific, Inc.) was carefully pipetted over each membrane. Finally, the membranes were visualized using a BioSpectrum ${ }^{\circledR}$ Imaging System (Ultra Violet Products, Ltd.). 
A
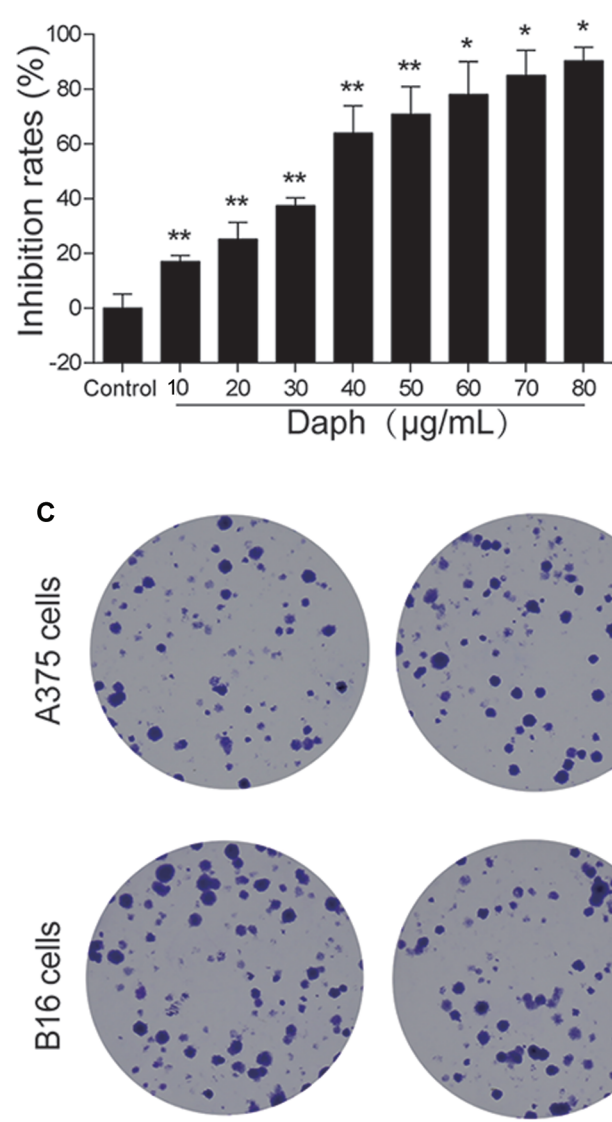

Control
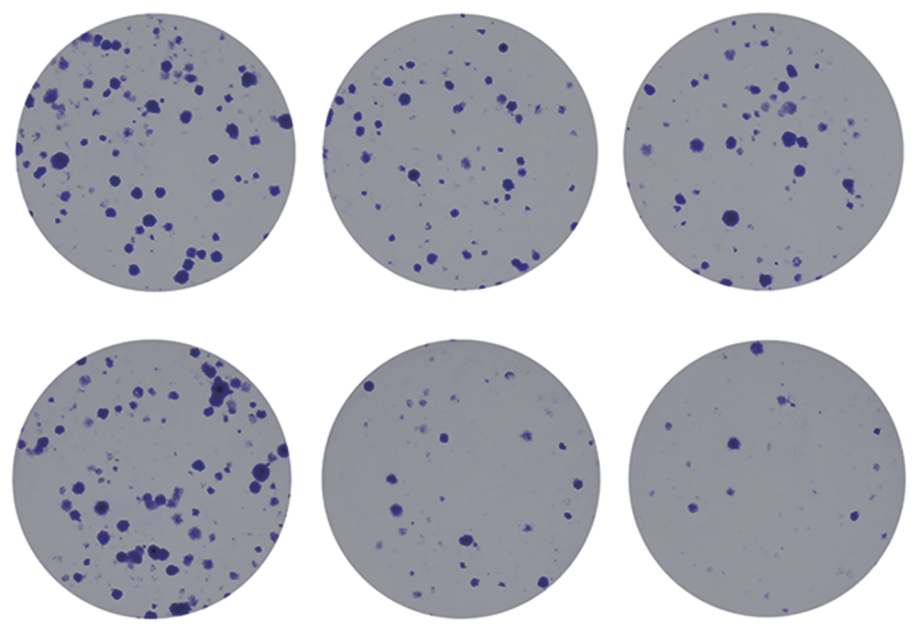

20

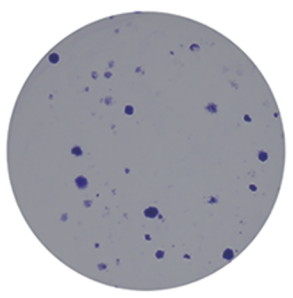

40
B

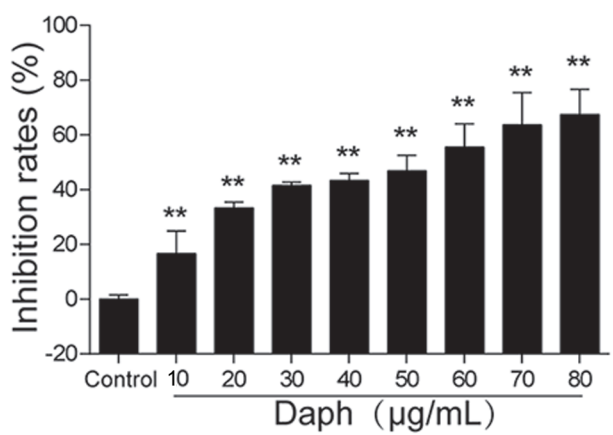

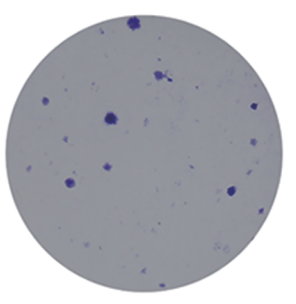

60

Daph $(\mu \mathrm{g} / \mathrm{mL})$

Figure 1. Daphnoretin inhibits the proliferation and colony-formation capacity of melanoma cells. MTT was used to determine the effects of different concentrations of daphnoretin on (A) A375 and (B) B16 cells after 24 h; cell viability of A375 or B16 cells. (C) Colonies were stained with crystal violet and observed under a microscope. Data are expressed as the mean $\pm \mathrm{SD}$ of three independent experiments. ${ }^{*} \mathrm{P}<0.05$ and ${ }^{* *} \mathrm{P}<0.01$ vs. control. Daph, daphnoretin.

ImageJ v1.43 software (National Institutes of Health) was used for densitometry.

Statistical analysis. The data are presented as the mean \pm standard deviation, and each experiment was repeated at least three times. One-way ANOVA or Student's t-test followed by Bonferroni's post hoc test were performed to compare the differences between groups using GraphPad Prism 6.0 (GraphPad Software, Inc.). P $<0.05$ was considered to indicate a statistically significant difference.

\section{Results}

Daphnoretin inhibits A375 and B16 cell proliferation and colony formation. An MTT assay was performed to evaluate the antitumor effect of daphnoretin in melanoma A375 and B16 cells. A 24-h treatment with daphnoretin significantly suppressed cellular proliferation, and the $\mathrm{IC}_{50}$ values for A 375 and B16 cells were 37.81 and $53.46 \mu \mathrm{g} / \mathrm{ml}$, respectively (Fig. 1A and B). Based on the results of the previous experiment, a colony formation assay was conducted; the results suggested that the number of A375 and B16 cell colonies gradually decreased compared with that of the control group
(Fig. 1C). These results indicated that daphnoretin inhibited cell proliferation.

Daphnoretin induces $A 375$ and B16 cell apoptosis. Hoechst 33258 staining was conducted to assess A375 and B16 cell apoptosis. Under a fluorescence microscope, the formation of apoptotic bodies was observed (Fig. 2A and D). To determine the mechanism of apoptosis in A375 and B16 cells following daphnoretin treatment, flow cytometry was used to quantify cells positive for annexin V-FITC and PI. Daphnoretin significantly increased the apoptotic rate of A375 and B16 cells in a dose-dependent manner (Fig. 2B and E). Moreover, the apoptotic rates of daphnoretin-treated A375 and B16 cells were significantly increased compared with those of the corresponding control groups (Fig. $2 \mathrm{C}$ and F). These findings suggested that daphnoretin induced apoptosis in A375 and B16 melanoma cells.

Daphnoretin induces melanoma cell apoptosis in a caspase-dependent manner. The effects of daphnoretin on the levels of apoptosis-related proteins were evaluated using A375 and B16 cells. Western blotting revealed that the protein levels of caspase-3 and -9 were both significantly decreased, 


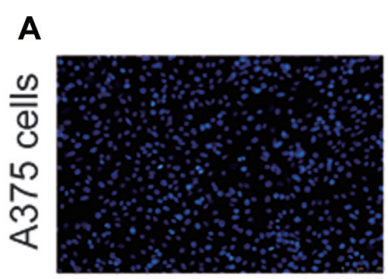

Control

B

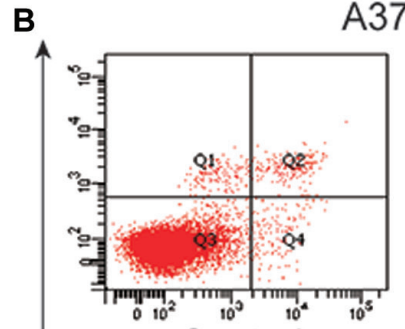

$\bar{\alpha}$

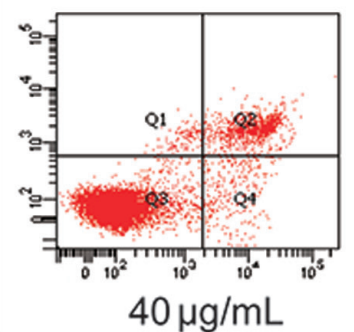

Annexin V-FITC

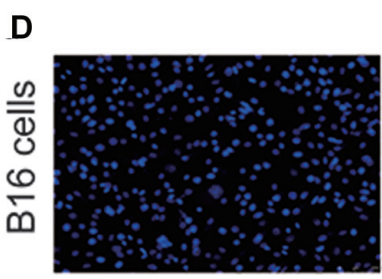

Control

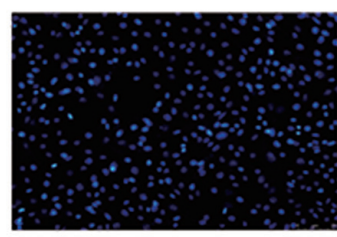

$20 \mu \mathrm{g} / \mathrm{mL}$

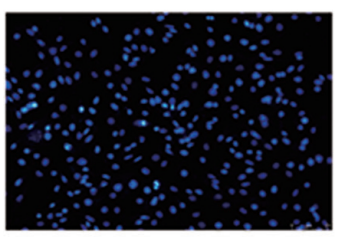

$40 \mu \mathrm{g} / \mathrm{mL}$

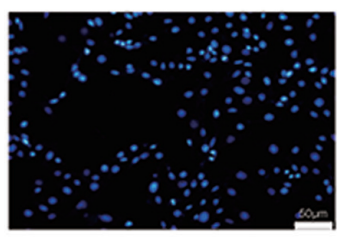

$60 \mu \mathrm{g} / \mathrm{mL}$

C

A375 cells
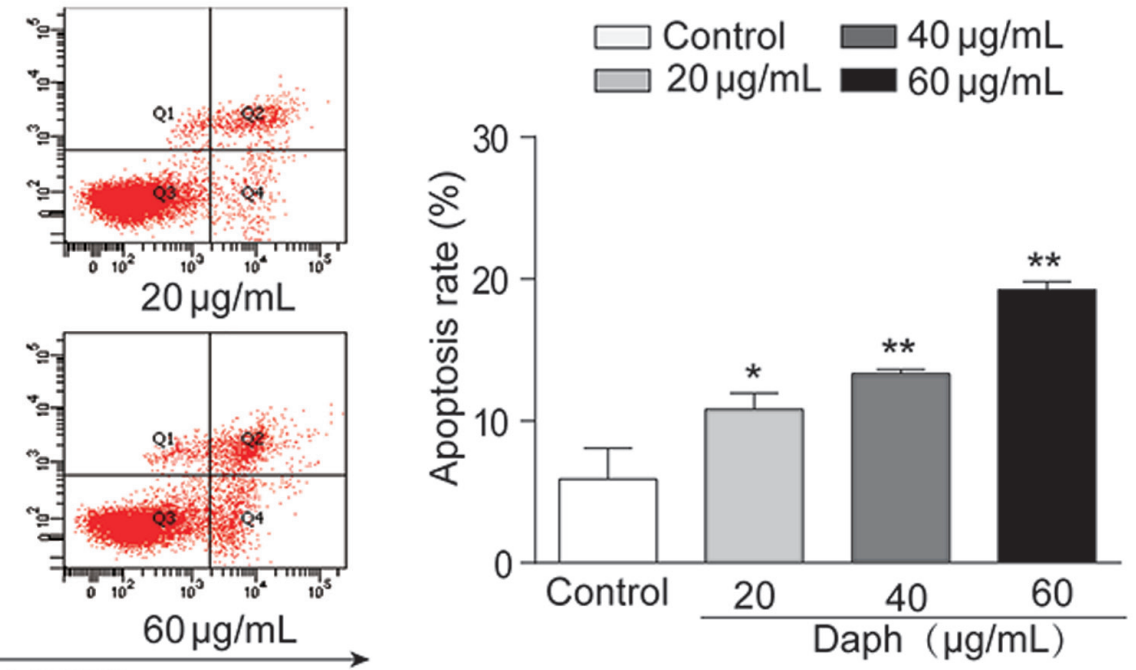

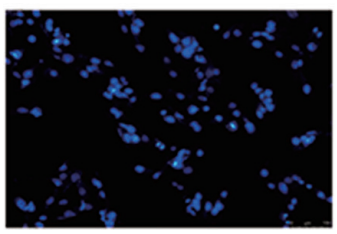

$40 \mu \mathrm{g} / \mathrm{mL}$

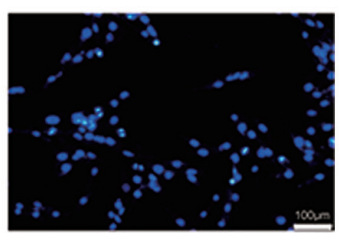

$60 \mu \mathrm{g} / \mathrm{mL}$

F

B16 cells

E

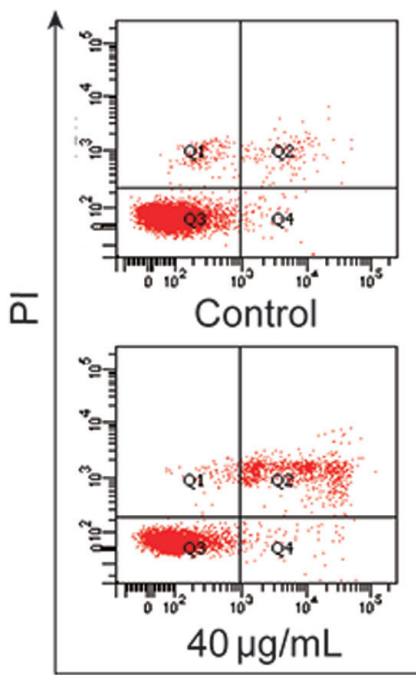

B16 cells

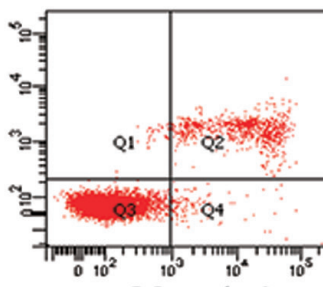

$20 \mu \mathrm{g} / \mathrm{mL}$

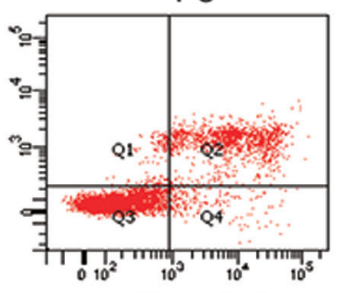

$60 \mu \mathrm{g} / \mathrm{mL}$

Annexin V-FITC

Figure 2. Daphnoretin induces A375 and B16 melanoma cell apoptosis. (A) Morphological changes in daphnoretin-treated A375 cells by Hoechst 33258 staining. (B) Apoptotic rate of daphnoretin-treated A375 cells was determined by flow cytometry. (C) Statistical analysis of the apoptosis rate of A375 cells after daphnoretin treatment. (D) Morphological changes in daphnoretin-treated B16 cells by Hoechst 33258 staining. (E) Apoptotic rate of daphnoretin-treated B16 cells was determined by flow cytometry. (F) Statistical analysis of the apoptosis rate of B16 cells after daphnoretin treatment. Data are expressed as the mean \pm SD of three independent experiments. ${ }^{*} \mathrm{P}<0.05$ and ${ }^{* *} \mathrm{P}<0.01$ vs. control. Daph, daphnoretin. 
A

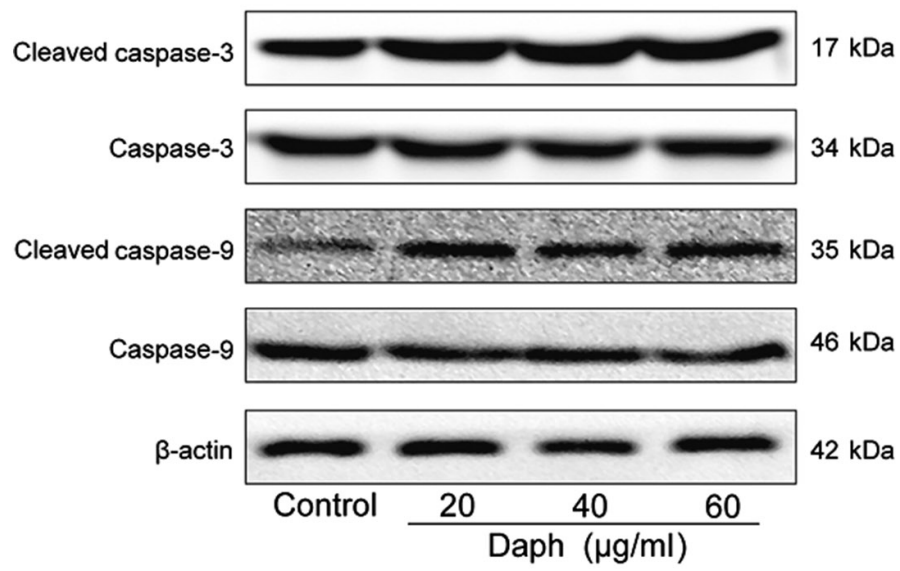

C

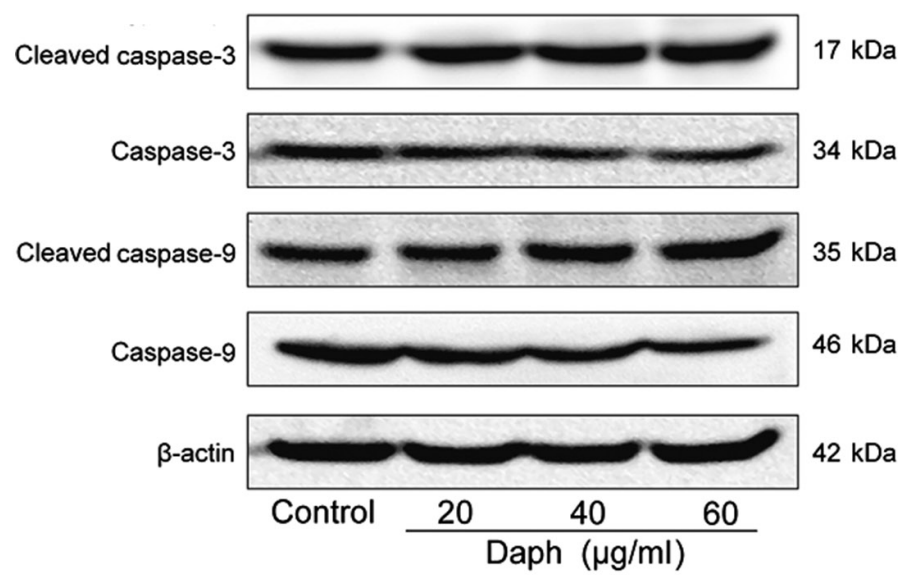

B

A375 cells

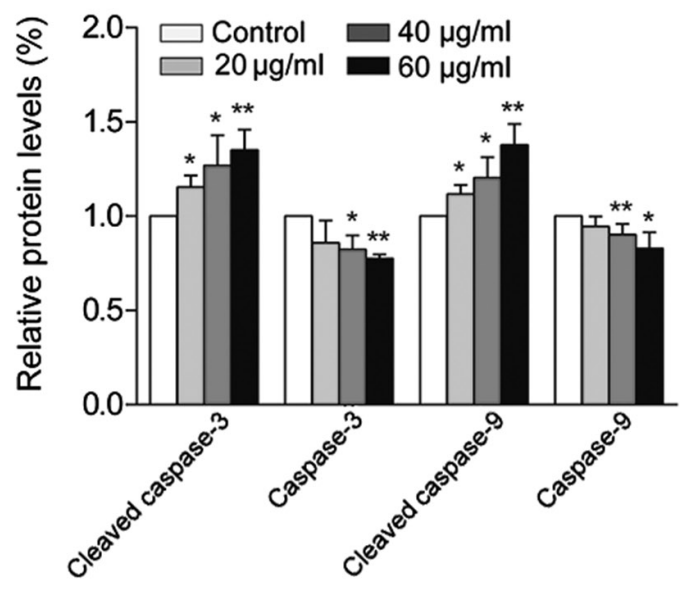

D

\section{B16 cells}

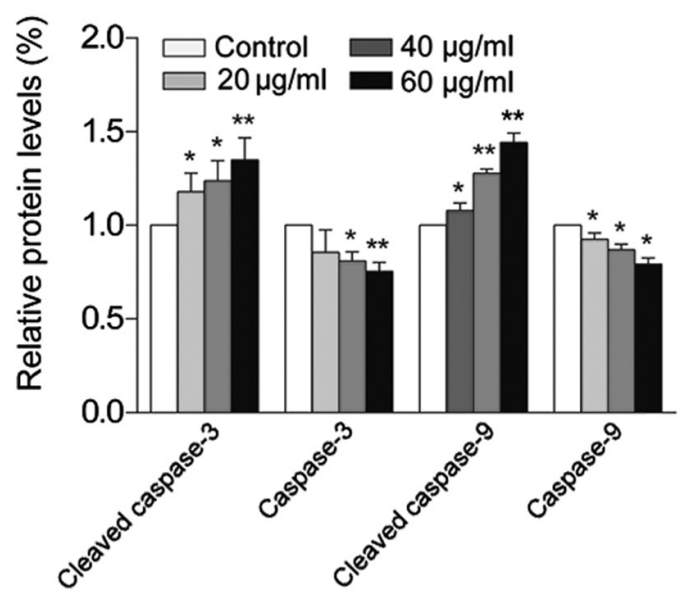

Figure 3. Daphnoretin induces melanoma cell apoptosis via a caspase-dependent mechanism. (A) Expression levels of cleaved caspase-3, caspase-3, cleaved caspase- 9 and caspase-9 in daphnoretin-treated A375 cells were measured by western blotting. (B) Statistical analysis of protein levels in A375 cells following daphnoretin treatment. (C) Expression levels of cleaved caspase-3, caspase-3, cleaved caspase-9 and caspase-9 in daphnoretin-treated B16 cells were quantified by western blotting. (D) Statistical analysis of the protein levels after daphnoretin treatment. Data are expressed as the mean \pm SD of three independent experiments. ${ }^{*} \mathrm{P}<0.05$ and ${ }^{* *} \mathrm{P}<0.01$ vs. control. Daph, daphnoretin.

while those of cleaved caspase- 3 and -9 were dose-dependently increased in daphnoretin-treated A375 cells (Fig. 3A and B). Similarly, the expression levels of caspase-3 and -9 were upregulated, and those of cleaved caspase- 3 and -9 were downregulated in daphnoretin-treated B16 cells, compared with the control group (Fig. 3C and D). These results suggested that daphnoretin induced apoptosis in A375 and B16 cells by a caspase-dependent mechanism.

Daphnoretin induces melanoma cell apoptosis via the $\mathrm{Bax} / \mathrm{BCl}-2$ pathway. The expression levels of another set of apoptosis-related proteins were measured by western blotting. The data showed that the levels of Bcl-2 were upregulated, whereas Bax, cytochrome $c$ and Apaf-1 were downregulated in daphnoretin-treated A375 cells (Fig. 4A and B). Similarly, the levels of Bcl-2 increased, while those of Bax, cytochrome $c$ and Apaf-1 decreased in B16 cells following treatment with daphnoretin (Fig. 4C and D). These results suggested that daphnoretin induced apoptosis in A375 and B16 cells by a Bax/Bcl-2-dependent pathway.

Daphnoretin increases the level of ROS production in melanoma cells. ROS plays a regulatory role in the process of apoptosis induced by various antitumor drugs (19). It has been confirmed that the increase in ROS may induce apoptosis (20). In the present study, the level DCF-associated green fluorescence was gradually increased in A 375 and B16 cells exposed to daphnoretin (Fig. 5A and C). In addition, the flow cytometry results showed that $24 \mathrm{~h}$ after daphnoretin treatment, the level of ROS in A375 and B16 cells dose-dependently increased compared with the corresponding control group (Fig. 5B and D). The data indicated that daphnoretin increased the level of ROS in melanoma cells.

Daphnoretin regulates the PI3K/Akt signaling pathway in A375 and B16 cells. The PI3K/Akt signaling pathway is an 

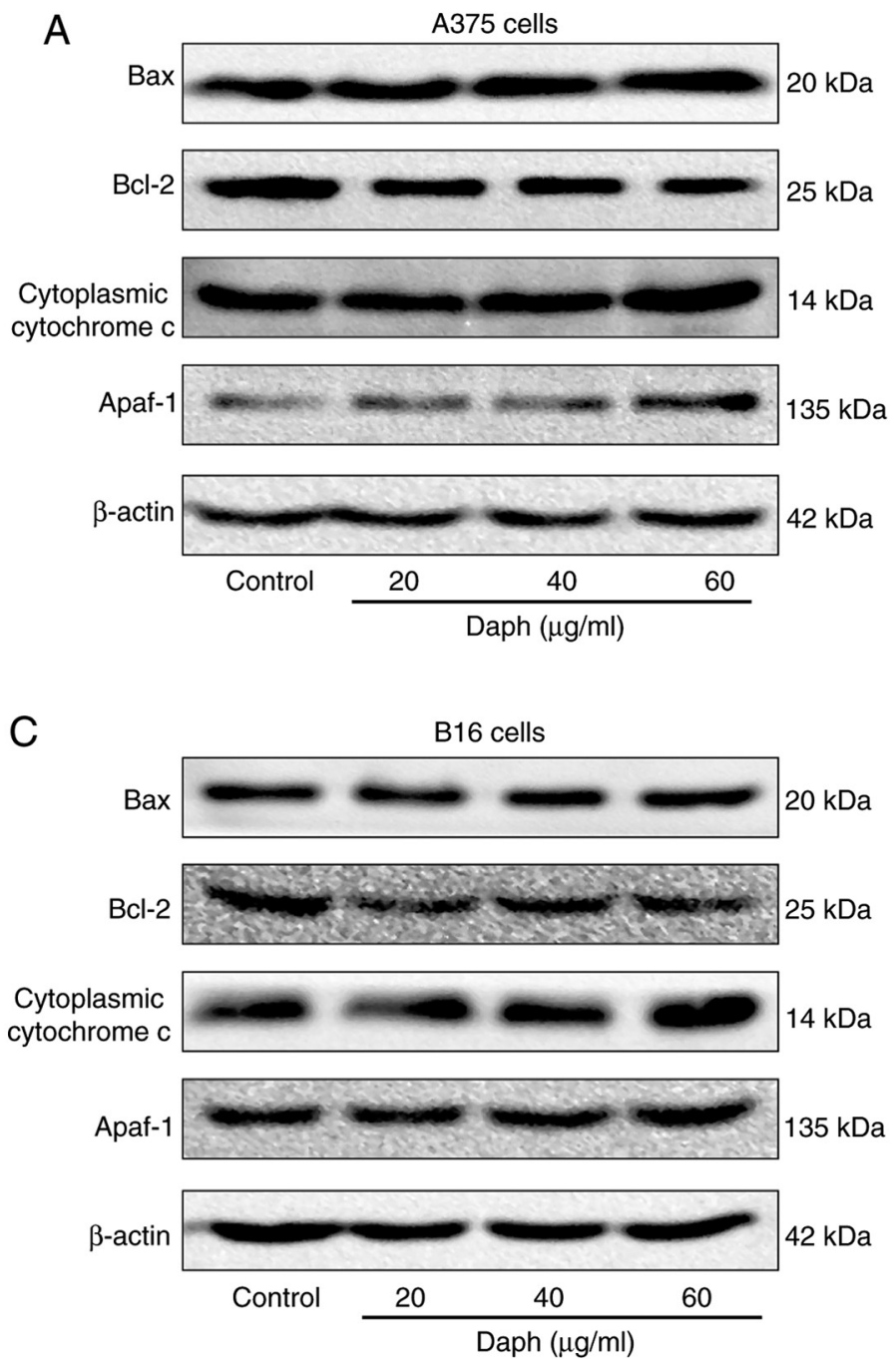

B

A375 cells

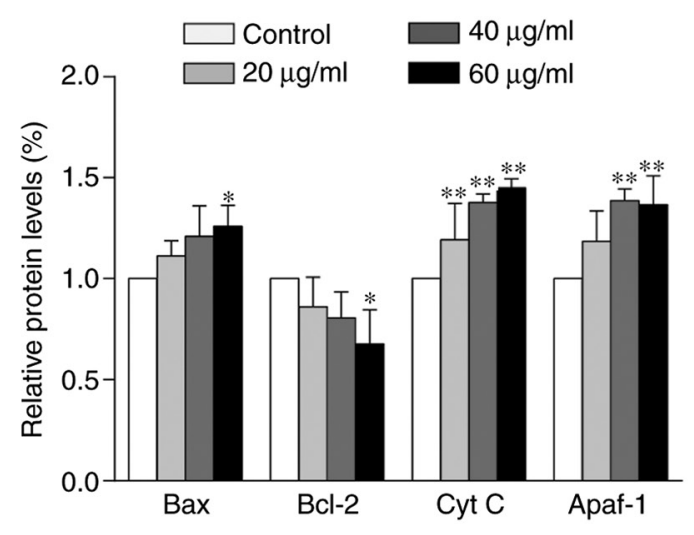

D B16 cells

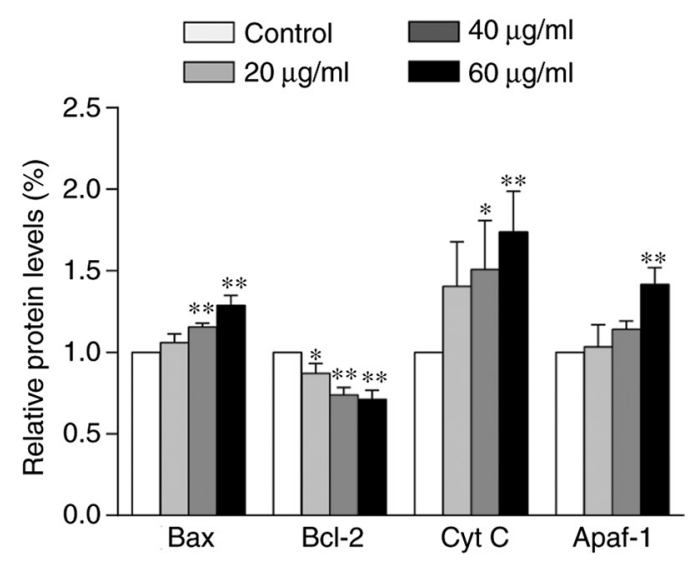

Figure 4. Daphnoretin induces melanoma cell apoptosis via a Bax/Bcl-2 dependent pathway. (A) Expression levels of Bax, Bcl-2, cytoplasmic cytochrome c and Apaf-1 in daphnoretin-treated A375 cells were measured by western blotting. (B) Statistical analysis of the protein levels of Bax, Bcl-2, cytoplasmic cytochrome c and Apaf-1 in A375 cells after daphnoretin treatment. (C) Expression levels of Bax, Bcl-2, cytoplasmic cytochrome c and Apaf-1 in daphnoretin-treated B16 cells were quantified by western blotting. (D) Statistical analysis of the protein levels after daphnoretin treatment. Data are expressed as the mean $\pm \mathrm{SD}$ of three independent experiments. $\mathrm{P}<0.05$ and ${ }^{* * *} \mathrm{P}<0.01$ vs. control. Daph, daphnoretin; Cyt $\mathrm{C}$, cytochrome c; Apaf-1, apoptotic protease-activating factor 1 .

important pathway closely related to tumor genesis and development, thus the protein levels of PI3K/Akt were assessed by western blotting. The results showed that the protein expression levels of PI3K and Akt were increased, whereas p-PI3K and p-Akt were decreased in daphnoretin-treated A375 and B16 cells (Fig. 6A-D). These results indicated that daphnoretin regulated the PI3K/Akt signaling pathway.

\section{Discussion}

Daphnoretin exerts antitumor effects in specific tumor types. A study demonstrated that daphnoretin induced apoptosis in human osteosarcoma cells by triggering $\mathrm{G}_{2} / \mathrm{M}$ cell cycle arrest and activating the caspase-3 pathway (21). In another study, daphnoretin was shown to induce the mitochondria-mediated apoptosis of HeLa cells (22). Daphnoretin has also been reported to influence the proliferation and apoptosis of A549 lung cancer cells in vitro (23). Moreover, daphnoretin was found to inhibit the proliferation, invasiveness and migration of colon cancer HCT116 cells, and to promote apoptosis by regulating Akt signaling pathway activity (24). The results of the present study demonstrated that daphnoretin inhibited proliferation and promoted apoptosis via the ROS-mediated mitochondrial apoptosis and the PI3K/Akt signaling pathways.

In the present study, daphnoretin was first found to significantly inhibit the proliferation of A375 and B16 melanoma cells in a concentration-dependent manner. To further confirm the mechanisms associated with the daphnoretin treatment, the formation of apoptotic bodies was observed in A375 and B16 cells following Hoechst staining, and the cellular apoptotic rate was significantly increased compared with the control group. These findings suggest that daphnoretin induced apoptosis in melanoma A375 and B16 cells.

Apoptosis is a complex process in which various protein signals are transmitted through several different pathways (25). Caspases, which are the convergence points of multiple apoptotic pathways, regulate the final execution of apoptosis $(26,27)$. Thus, caspase activation is an essential link 


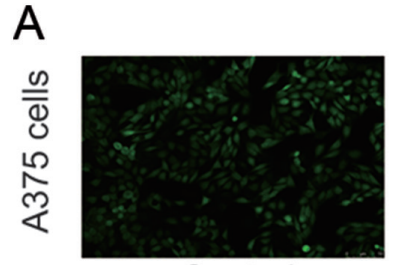

Control

B

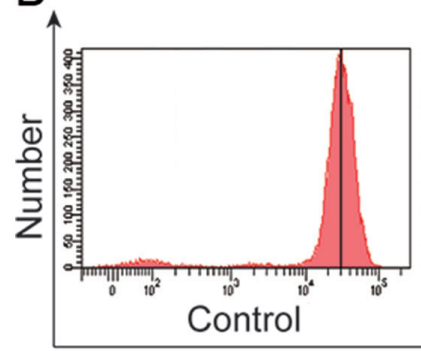

C

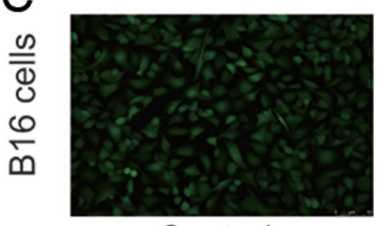

Control

D

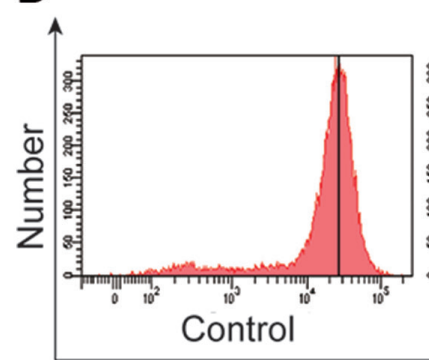

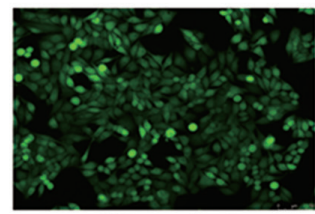

$20 \mu \mathrm{g} / \mathrm{ml}$

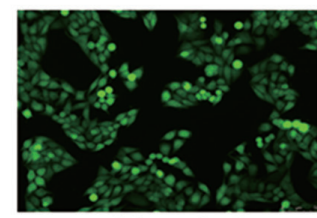

$40 \mu \mathrm{g} / \mathrm{ml}$

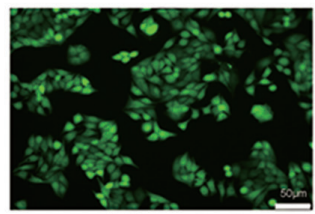

$60 \mu \mathrm{g} / \mathrm{ml}$

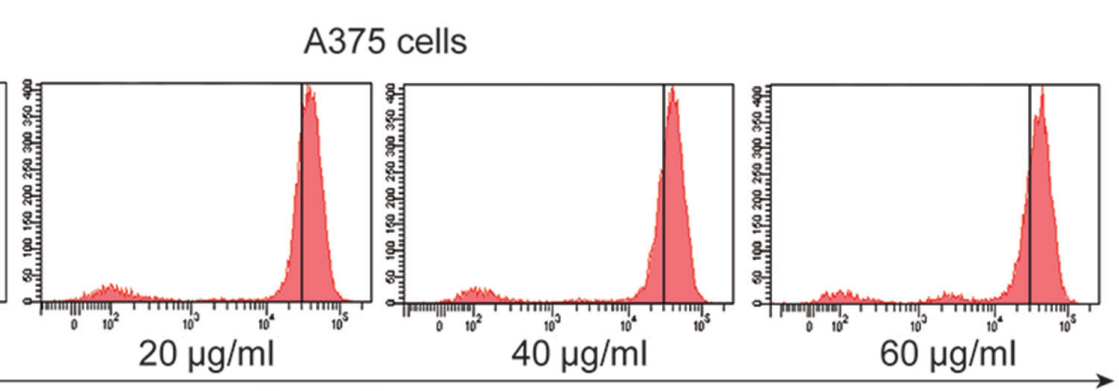

Green fluorescence

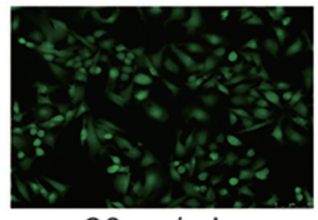

$20 \mu \mathrm{g} / \mathrm{ml}$

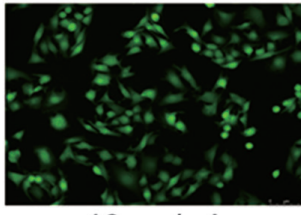

$40 \mu \mathrm{g} / \mathrm{ml}$

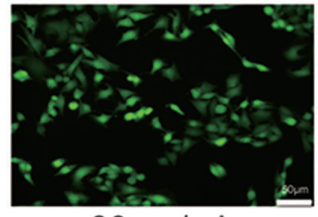

$60 \mu \mathrm{g} / \mathrm{ml}$

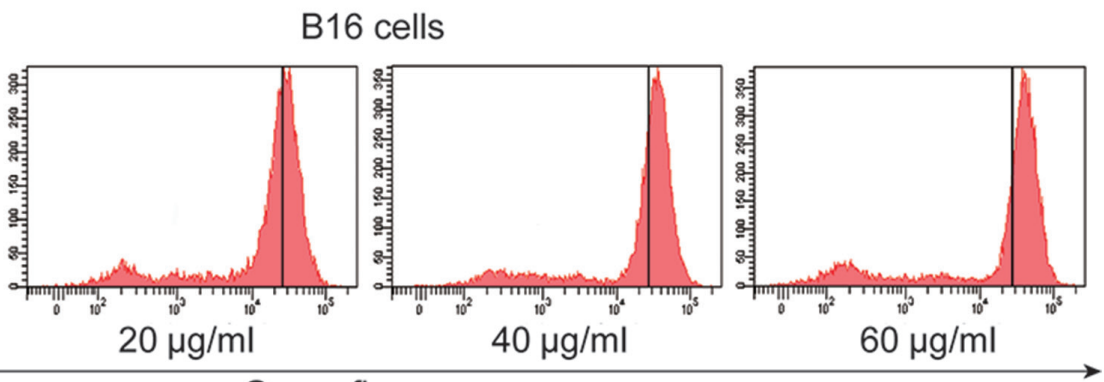

Green fluorescence

Figure 5. Daphnoretin induces ROS-mediated apoptosis in melanoma A375 and B16 cells. (A) Morphological changes in daphnoretin-treated A375 cells. (B) ROS content of daphnoretin-treated A375 cells was measured by flow cytometry. (C) Morphological changes in daphnoretin-treated B16 cells. (D) ROS content of daphnoretin-treated B16 cells was measured by flow cytometry. ROS, reactive oxygen species.

in apoptotic signal transduction (28). Under the stimulation of apoptotic signals, cytochrome $c$ is released into the cytoplasm and combines with Apaf-1 to activate the caspase-9 precursor; activated caspase- 9 then cleaves the caspase- 3 proenzyme, which activates caspase-3, thus promoting its cleavage. Finally, a caspase cascade reaction is triggered, and apoptosis is induced $(29,30)$. The present findings indicate that the levels of caspase- 3 and -9 were significantly decreased, whereas the levels of cleaved caspase-3 and -9 were markedly increased compared with the control group.

Several studies have suggested that the Bcl-2 family plays a vital role in the apoptotic process $(31,32)$. The expression and regulation of Bcl-2 family proteins are essential in signal transduction pathways and apoptosis. The pro-apoptotic protein Bax, and the anti-apoptotic protein Bcl-2, are essential members of the Bcl-2 family, and their coordinated action plays an important role in apoptosis (33). Bcl-2 and Bax usually exist as heterodimers, which jointly regulate apoptosis $(34,35)$. When the expression of $\mathrm{Bcl}-2$ is high, a large number of heterodimers with
Bax are formed, inhibiting the activity of Bax and preventing apoptosis; when the expression of Bax exceeds a certain point, Bax homodimers predominate, which induces apoptosis $(35,36)$. The present results showed that the expression levels of Bax increased, while those of Bcl-2 decreased, suggesting that the Bcl-2 family regulates daphnoretin-induced apoptosis. Previous studies have shown that the Bcl-2 family members release cytochrome $c$ and activated various caspases $(37,38)$. Therefore, the results of the present study indicate that daphnoretin induces the activity of the Bcl-2 family proteins, and promote the release of cytochrome $c$ and Apaf-1, thereby activating caspase-9 and -3, and ultimately inducing apoptosis.

An impairment of the ROS balance is associated with cancer cell proliferation inhibition. Compared with normal untransformed cells, ROS levels are increased in cancer cells (39). As signaling molecules in cellular regulation, ROS play an important role in apoptosis by regulating apoptotic factors (40). Deoxynivalenol induces the cytotoxicity of IPEC-J2 cells in a dose-dependent manner, in 

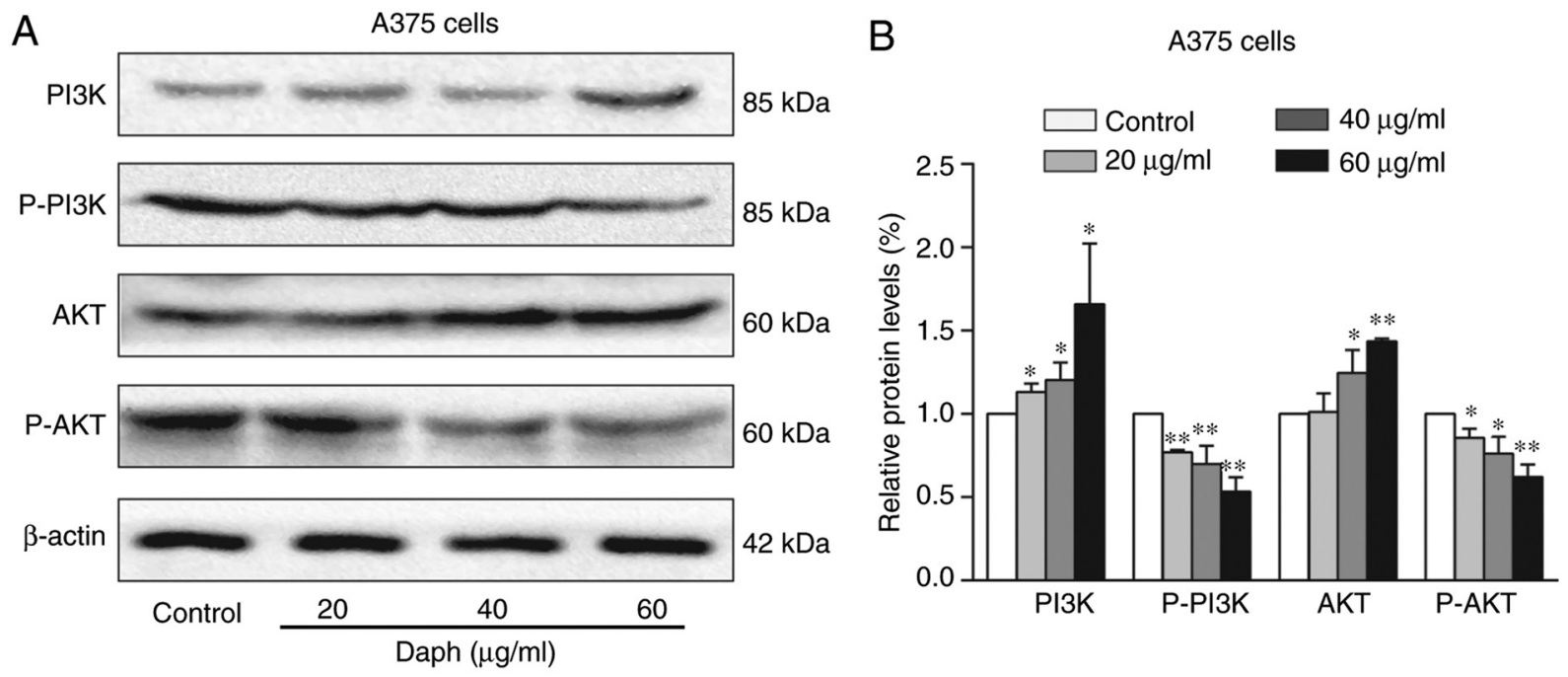

C
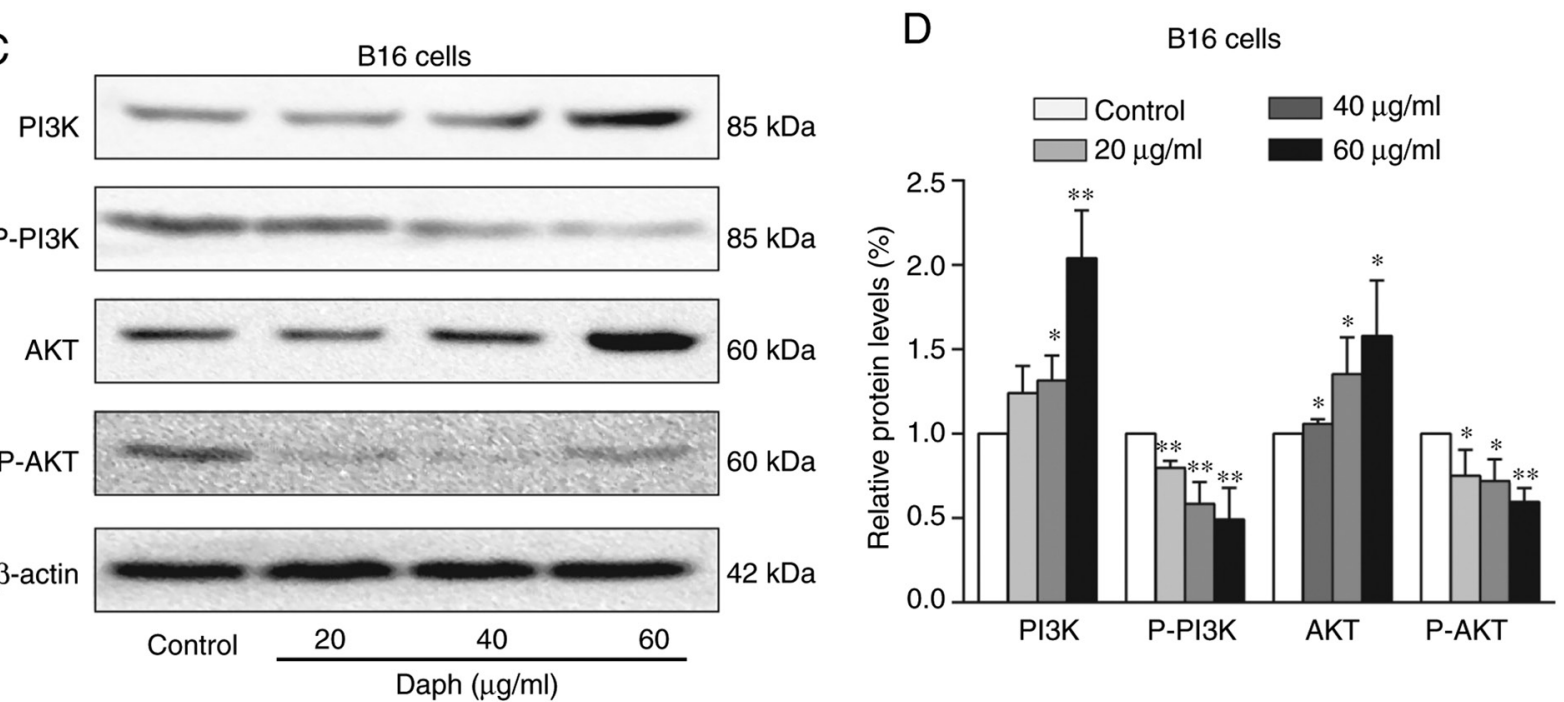

Figure 6. Daphnoretin regulates the PI3K/Akt signaling pathway in A375 and B16 cells. (A) Levels of PI3K, p-PI3K, Akt and p-Akt in daphnoretin-treated A375 cells were measured by western blotting. (B) Statistical analysis of the protein levels in A375 cells following daphnoretin treatment. (C) Levels of PI3K, p-PI3K, Akt and p-Akt in daphnoretin-treated B16 cells were assessed by western blotting. (D) Statistical analysis of the protein levels in daphnoretin-treated B16 cells. Data are expressed as the mean \pm SD of three independent experiments. ${ }^{*} \mathrm{P}<0.05$ and ${ }^{* *} \mathrm{P}<0.01$ vs. control. Daph, daphnoretin; $\mathrm{p}-$, phosphorylated.

which apoptosis is potentially the result of increased ROS production (41). After cells receive pro-apoptotic signals, ROS generation increases, which promotes $\mathrm{Ca}^{2+}$ influx, upregulates the expression of Bax, initiates mitochondrial permeability transition pore formation, and activates caspases, which ultimately leads to apoptosis. A previous study revealed that ROS-induced apoptosis may be related to the ROS-dependent upregulation of Bax, and a reduction in $\mathrm{Bcl}-2$ expression $(42,43)$. Another study indicated that the activation of caspase- 3 was regulated by ROS (44). To investigate these mechanisms in A375 and B16 cells treated with daphnoretin, ROS generation was measured by flow cytometry, which revealed increased levels of ROS in A375 and B16 cells. These results indicated that daphnoretin induced ROS-mediated apoptosis in melanoma A375 and B16 cells.

ROS have also been shown to play important roles in various signaling pathways, including the PI3K/Akt, Wnt $/ \beta$-catenin and MAPK pathways (45). It has been reported that genistein induces $\mathrm{G}_{2} / \mathrm{M}$ cell cycle arrest and apoptosis in human bladder transitional cell carcinoma T24 cells through the ROS-mediated PI3K/Akt signaling pathway (46), which is also important for regulating ROS generation in HCT116 cells (47). Therefore, the potential association between PI3K/Akt and the daphnoretin-induced inhibition of proliferation and increased apoptosis were investigated using A375 and B16 cells. The findings suggested that the levels of PI3K and Akt increased, and that those of p-PI3K and p-Akt decreased, in daphnoretin-treated A375 and B16 cells, indicating that the PI3K/Akt signaling pathway is involved in the antitumor effect of daphnoretin in melanoma cells.

In conclusion, daphnoretin markedly inhibited cellular proliferation and induced apoptosis through the ROS-mediated mitochondrial apoptosis and PI3K/Akt signaling pathways in melanoma A375 and B16 cells. These findings demonstrate that daphnoretin has the potential to be used as an anti-melanoma drug. However, the future clinical application of daphnoretin requires further investigation. 


\section{Acknowledgements}

Not applicable.

\section{Funding}

The present study was funded by the National Natural Science Foundation of China (grant no. 31870338), the Key Research and Development Program of Shandong Province of China (grant no. 2019GSF108214), Taishan Scholars Construction Engineering of Shandong Province (grant no. tsqn201812099), the Dominant Disciplines' Talent Team Development Scheme of Higher Education of Shandong Province (grant no. 2016052410) and the Introduction and Cultivation Project for Young Creative Talents of Higher Education of Shandong Province (grant no. 20191008198).

\section{Availability of data and materials}

All data generated or analyzed during this study are included in this published article.

\section{Authors' contributions}

$\mathrm{HW}, \mathrm{XH}, \mathrm{DL}$ and QZ designed the experiments. HW and $\mathrm{XH}$ obtained the experimental data and wrote the manuscript. HW, ML and ZP performed Hoechst 33258 staining, flow cytometric apoptosis analysis, western blot analysis and ROS detection. HW and DL interpreted and analyzed the data. HW and QZ assessed the authenticity of all the raw data. All authors read and approved the final manuscript.

\section{Ethics approval and consent to participate}

Not applicable.

\section{Patient consent for publication}

Not applicable.

\section{Competing interests}

The authors declare that they have no competing interests.

\section{References}

1. George Liddell H and Scott R: Drugs in clinical development for melanoma. Pharmaceut Med 26: 171-183, 2012.

2. Saavedra-Alonso S, Zapata-Benavides P, Chavez-Escamilla AK, Manilla-Muñoz E, Zamora-Avila DE, Franco-Molina MA and Rodriguez-Padilla C: WT1 shRNA delivery using transferrin-conjugated PEG liposomes in an in vivo model of melanoma. Exp Ther Med 12: 3778-3784, 2016.

3. Pippa C, Mirela H, Kate F and Christine P: Management of melanoma. Br Med Bull 111: 149-162, 2014.

4. Deiana M, Dalle Carbonare L, Serena M, Cheri S, Parolini F, Gandini A, Marchetto G, Innamorati G, Manfredi M, Marengo E, et al: New insights into the runt domain of RUNX2 in melanoma cell proliferation and migration. Cells 7: 220, 2018.

5. Chen L and Jin S: Trends in mortality rates of cutaneous melanoma in East Asian populations. PeerJ 4: e2809, 2016.

6. Wada-Ohno M, Ito T and Furue M: Adjuvant therapy for melanoma. Curr Treat Options Oncol 20: 63, 2019.
7. Tchernev G: One step melanoma surgery for patient with thick primary melanomas: 'To break the rules, you must first master them!' Open Access Maced J Med Sci 6: 367-371, 2018.

8. Hsu SC and Chung JG: Anticancer potential of emodin. Biomedicine 2: 108-116, 2012.

9. Sun X, Yan H, Zhang Y, Wang X, Qin D and Yu J: Preparative separation of diterpene lactones and flavones from Andrographis paniculate using off-line two-dimensional high-speed counter-current chromatography. Molecules 24: 620, 2019.

10. Mao W and Xia Q: Anti-tumor effects of traditional Chinese medicine give a promising perspective. J Cancer Res Ther 10 (Suppl 1): S1-S2, 2014.

11. Luo F, Gu J, Chen L and Xu X: Systems pharmacology strategies for anticancer drug discovery based on natural products. Mol Biosyst 10: 1912-1917, 2014.

12. Zhaokun Y, Zijun L and Jiumao L: Anticancer properties of traditional Chinese medicine. Comb Chem High Throughput Screen 20: 423-429, 2017.

13. Xiang Y, Guo Z, Zhu P, Chen J and Huang Y: Traditional Chinese medicine as a cancer treatment: Modern perspectives of ancient but advanced science. Cancer Med 8: 1958-1975, 2019.

14. Chen CA, Liu CK, Hsu ML, Chi CW, Ko CC, Chen JS, Lai CT, Chang HH, Lee TY, Lai YL and Chen YJ: Daphnoretin modulates differentiation and maturation of human dendritic cells through down-regulation of c-Jun N-terminal kinase. Int Immunopharmacol 51: 25-30, 2017.

15. Lu CL, Li YM, Fu GQ, Yang L, Jiang JG, Zhu L, Lin FL, Chen J and Lin QS: Extraction optimisation of daphnoretin from root bark of Wikstroemia indica (L.) C.A. and its anti-tumour activity tests. Food Chem 124: 1500-1506, 2011.

16. Van MJ, Kaspers GJ and Cloos J: Cell sensitivity Assays: The MTT assay. Methods Mol Biol 88: 237-245, 2011.

17. Wu S, Zhu G, Ni Y, Zhang T and Jiang W: Cucurbitacin I (JSI-124)-induced apoptosis of HepG2 cells via p53 signaling pathway. Xi Bao Yu Fen Zi Mian Yi Xue Za Zhi 33: 33-38, 2017 (In Chinese).

18. Yurinskaya V, Aksenov N, Moshkov A, Model M, Goryachaya T and Vereninov A: A comparative study of U937 cell size changes during apoptosis initiation by flow cytometry, light scattering, water assay and electronic sizing. Apoptosis 22: 1287-1295, 2017.

19. Luu HN, Wen W, Li H, Dai Q, Yang G, Cai Q, Xiang YB, Gao YT, Zheng W and Shu XO: Are dietary antioxidant intake indices correlated to oxidative stress and inflammatory marker levels? Antioxid Redox Signal 22: 951-959, 2015.

20. Zhang B, Peng X, Li G, Xu Y, Xia X and Wang Q: Oxidative stress is involved in Patulin induced apoptosis in HEK293 cells. Toxicon 94: 1-7, 2015.

21. Gu S and He J: Daphnoretin induces cell cycle arrest and apoptosis in human osteosarcoma (HOS) cells. Molecules 17: 598-612, 2012.

22. Yang ZY, Kan JT, Cheng ZY, Wang XL, Zhu YZ and Guo W: Daphnoretin-induced apoptosis in HeLa cells: A possible mitochondria-dependent pathway. Cytotechnology 66: 51, 2014.

23. Jiang HF, Wu Z, Bai X, Zhang Y and He P: Effect of daphnoretin on the proliferation and apoptosis of A549 lung cancer cells in vitro. Oncol Lett 8: 1139-1142, 2014.

24. Yu S, Guo H, Gao X, Li M and Bian H: Daphnoretin: An invasion inhibitor and apoptosis accelerator for colon cancer cells by regulating the Akt signal pathway. Biomed Pharmacother 111: 1013-1021, 2019.

25. Olechowska-Jarząb A, Ptak-Belowska A and Brzozowski T: Terapeutic importance of apoptosis pathways in pancreatic cancer. Folia Med Cracov 56: 61-70, 2016.

26. Li D, Hu X, Han T, Liao J, Xiao W, Xu S, Li Z, Wang Z, Hua H and $\mathrm{Xu}$ J: NO-releasing enmein-type diterpenoid derivatives with selective antiproliferative activity and effects on apoptosis-related proteins. Molecules 21: 1193, 2016.

27. Lkhagvasuren $\mathrm{K}$ and Kim JK: Ziyuglycoside II induces caspases-dependent and caspases-independent apoptosis in human colon cancer cells. Toxicol In Vitro 59: 255-262, 2019.

28. Palai TK and Mishra SR: Caspases: An apoptosis mediator. J Adv Veterinary Animal Res 2: 18-22, 2015.

29. Frejlich E, Rudno-Rudzińska J, Janiszewski K, Salomon L and Kielan W: Caspases and their role in gastric cancer. Adv Clin Exp Med 22: 593-602, 2013.

30. Zhao Y, Jing Z, Lv J, Zhang Z, Lin J, Cao X, Zhao Z, Liu P and Mao W: Berberine activates caspase-9/cytochrome $c$ - mediated apoptosis to suppress triple-negative breast cancer cells in vitro and in vivo. Biomed Pharmacother 95: 18-24, 2017. 
31. Sheng M, Zhou Y, Yu W, Weng Y, Xu R and Du H: Protective effect of Berberine pretreatment in hepatic ischemia/ reperfusion injury of rat. Transplant Proc 47: 275-282, 2015.

32. Kvansakul M and Hinds MG: The Bcl-2 family: Structures, interactions and targets for drug discovery. Apoptosis 20: 136-150, 2015.

33. Galluzzi L, Kepp O, Trojel-Hansen C and Kroemer G: Mitochondrial control of cellular life, stress, and death. Circ Res 111: 1198, 2012.

34. Sawa A and Sedlak TW: Oxidative stress and inflammation in schizophrenia. Schizophr Res 176: 1-2, 2016

35. Jin S and Dai CL: Attenuation of reperfusion-induced hepatocyte apoptosis is associated with reversed bcl-2/bax ratio in hemi-hepatic artery-preserved portal occlusion. J Surg Res 174: 298-304, 2012

36. Ding H, Wang J, Jia FP, Yi J and Zhang M: Research on the A549 cell apoptosis mechanism of the nude mouse model using MenSC-sTRAIL. Eur Rev Med Pharmacol Sci 21: 3218-3222, 2017.

37. Burrer CM, Foight GW, Keating AE and Chan GC: Selective peptide inhibitors of antiapoptotic cellular and viral Bcl-2 proteins lead to cytochrome $c$ release during latent Kaposi's sarcoma-associated herpesvirus infection. Virus Res 211: 86-88, 2016.

38. Gupta R and Ghosh S: Putative roles of mitochondrial Voltage-dependent anion channel, Bcl-2 family proteins and c-Jun N-terminal Kinases in ischemic stroke associated apoptosis. Biochimie Open 4: 47-55, 2017.

39. Jutooru I, Guthrie AS, Chadalapaka G, Pathi S, Kim K, Burghardt R, Jin UH and Safe S: Mechanism of action of phenethylisothiocyanate and other reactive oxygen species-inducing anticancer agents. Mol Cell Biol 34: 2382-2395, 2014.

40. Hong M, Li J, Li S and Almutairi MM: Acetylshikonin sensitizes hepatocellular carcinoma cells to apoptosis through ROS-mediated caspase activation. Cells 8: 1466, 2019.
41. Kang R, Li R,Dai P,Li Z,Li Y and Li C: Deoxynivalenol induced apoptosis and inflammation of IPEC-J2 cells by promoting ROS production. Environ Pollut 251: 689-698, 2019.

42. Choi YH, Kang YJ, Kim SH, Sung B and Kim ND: Abstract 1773: MHY-449 induces apoptotic cell death through ROS- and caspase-dependent pathways in AGS human gastric cancer cells. Cancer Res 75: 1773, 2015.

43. AlBasher G, AlKahtane AA, Alarifi S, Ali D, Alessia MS, Almeer RS, Abdel-Daim MM, Al-Sultan NK, Al-Qahtani AA, AliH and Alkahtani S: Methotrexate-induced apoptosis in human ovarian adenocarcinoma SKOV-3 cells via ROS-mediated bax/bcl-2-cyt-c release cascading. Onco Targets Ther 12: 21-30, 2018.

44. Chen L, Gong MW, Peng ZF, Zhou T, Ying MG, Zheng QH, Liu QY and Zhang QQ: The marine fungal metabolite, dicitrinone B, induces A375 cell apoptosis through the ROS-related caspase pathway. Mar Drugs 12: 1939-1958, 2014.

45. Lee SY, Jeong EK, Ju MK, Jeon HM, Kim MY, Kim CH, Park HG, Han SI and Kang HS: Induction of metastasis, cancer stem cell phenotype, and oncogenic metabolism in cancer cells by ionizing radiation. Mol Cancer 16: 10, 2017

46. Park C, Cha HJ, Lee H, Hwang-Bo H, Ji SY, Kim MY, Hong SH, Jeong JW, Han MH, Choi SH, et al: Induction of G2/M cell cycle arrest and apoptosis by genistein in human bladder cancer T24 cells through inhibition of the ROS-dependent PI3k/Akt signal transduction pathway. Antioxidants (Basel) 8: 327, 2019.

47. Gao X, Li X, Ho CT, Lin X and Chen Z: Cocoa tea (Camellia ptilophylla) induces mitochondria-dependent apoptosis in HCT116 cells via ROS generation and PI3K/Akt signaling pathway. Food Res Int 129: 108854, 2019.

c) (i) $(-)$ This work is licensed under a Creative Commons

EY No No Attribution-NonCommercial-NoDerivatives 4.0 International (CC BY-NC-ND 4.0) License. 